\title{
The Importance of Being Active
}

\author{
Björn Brembs \\ Freie Universität Berlin, Institut für Biologie-Neurobiologie, Berlin, Germany
}

\begin{abstract}
The successful stimulus-response approach to the organization of behavior has been the dominating paradigm for much of the psychology and neuroscience of the 20th century. Martin Heisenberg is a pioneer in championing the idea that all brains, even comparatively simple ones such as those of insects, instead operate according to output-input principles. Since the 1970s, his research produces evidence that the fruit fly, Drosophila melanogaster, is capable of spontaneous behavioral activity, and that the flies use it to control sensory input (i.e., operant behavior). Today, more and more evidence is accumulating also from fields outside of neuroscience that, indeed, one of the common, defining principles of all brains is this concept of operant behavior. Drawing from this evidence, it becomes clear that the conceptually simple process of generating activity and evaluating its consequences forms one of the fundamental cornerstones not only for all of our human nature, but also for our social coherence. This review recapitulates Heisenberg's most critical experiments and provides an overview over the current literature on the role of spontaneous activity in the ecology and evolution of brains. I conclude that spontaneous activity is both a necessary prerequisite and an inevitable consequence of evolution.
\end{abstract}

Keywords: spontaneous activity, intiating activity, evolution, brain, Heisenberg

Probably one of the - if not the best-understood sensory system is the fly visual system. Over the last 60 years or so, on all levels of complexity, the systems, the single cell and, more recently, even the molecular level, neuroscientists have developed an understanding that is unparalleled both in breadth and in depth. The groundwork was laid by the early works of Götz, Reichardt, and other colleagues in the tradition of biological cybernetics (Götz, 1964, 1965, 1968, 1972, 1977, 1980; Götz and Buchner, 1978; Götz et al., 1979; Kirschfeld and Reichardt, 1970; Poggio and Reichardt, 1973a, 1973b; Poggio and Reichardt, 1976a, 1976b; Reichardt, 1962; Reichardt and Poggio, 1975, 1976; Reichardt and Wenking, 1969; Reichardt, 1965; Wehrhahn and Reichardt, 1973). This tradition entailed to study the visual system with the tools of control theory. In principle, this meant interpreting such experiments as manipulating a complex input-output system. The idea behind this very successful black-box approach was to study the input-output relationships thoroughly enough to be able to construct a control model that could predict the motor output of the fly for any, even yet untested, visual input. One method of choice was often the so-called open-loop experiment, in which the tethered fly (Figure 1) received visual input while its motor output was recorded. Importantly, the motor output was not allowed to interfere with the presentation of the stimuli (i.e., the feedback loop between the animal's behavior and its environment was open). This was the time when the young geneticist, Martin Heisenberg, joined this exciting field after his postdoctoral period with Seymour Benzer at CalTech. Heisenberg's contributions to vision in Drosophila are covered elsewhere in this issue, so I will not go into any detail here. This input-output approach worked extremely well, and not only for biological cybernetics of visual guidance of insect flight. Many other areas of neurobiology, at the time, also thought of brains as input-output systems and prevented the behavior of their animals to interfere with their stimulus situation. In fact, so successful was this pervasive approach that until very recently, some neurobiologists still emphasized that "brain function is ultimately best understood in terms of input/ output transformations and how they are produced" (Mauk, 2000). This was the dominant tradition in which Heisenberg was working when he moved to Würzburg to become the chair of the department of genetics in 1975 .

In Würzburg, several discoveries prompted Heisenberg to radically change his view on brains. Three of these, in particular, were instrumental for his 180-degree turn. First was the observation that even without any variation in sensory input, the flies would produce varying motor output (Figure 2; Heisenberg and Wolf,

Received 20 August 2008; Accepted 10 September 2008.

Address correspondence to Björn Brembs, Freie Universität Berlin, Institut für Biologie-Neurobiologie, Königin Luise Str. 28-30, Berlin 14195, Germany. E-mail: bjoem@brembs.net 


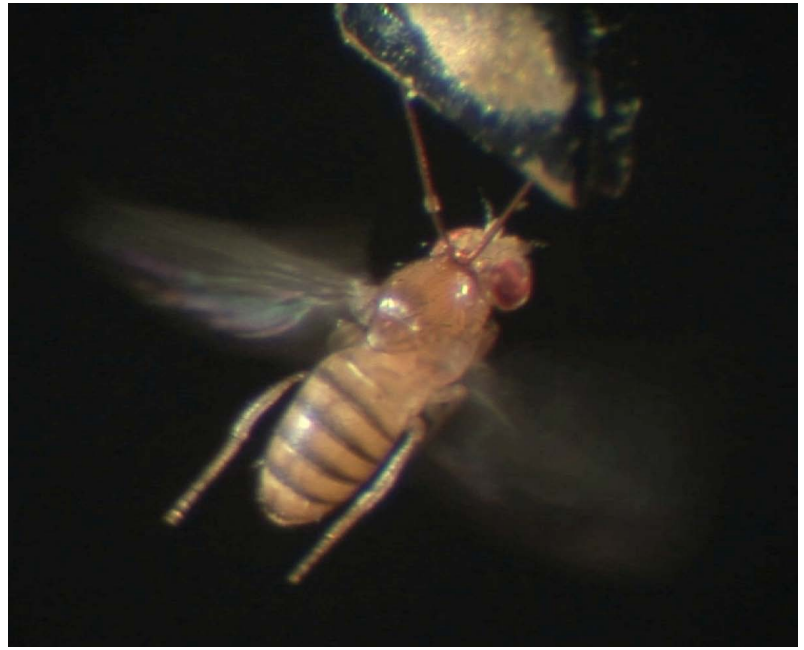

Figure 1. A Drosophila fruit fly, suspended at a copper hook, attached to the head and thorax of the fly by a small drop of glue.

1979). This finding flew in the face of every control theory thus far. A later mathematical analysis of the temporal structure of the flies' behavior in this situation (Maye et al., 2007) confirmed Heisenberg's interpretation that the variability in the behavior of the fly does not stem from a very noisy input-output system, but was generated actively by the fly, independently of any input (i.e., "initiating activity"; Heisenberg, 1983, 1994). Importantly, Maye et al. (2007) found the same kind of variability in the behavior even when the flies were allowed to control their environment with the feedback loop closed. The second discovery was made with the double-mutant, reduced optic lobes (rol), small optic lobes (sol) (Figure 3). Freely walking or flying wildype flies, in a visually structured rotating environment, have a tendency to turn with the direction of the movement. The rol sol double mutant flies still show phototaxis, but are completely devoid of any such directed "optomotor response." The optomotor response was thought to be critical for stabilizing the animal in flight, and thus rol sol flies were expected to lack the capacity to use moving visual stimuli for course control and thus should show unstable flight. However, in experiments with tethered flies where the feedback loop between turning behavior and horizontal rotation of the environment was closed, rol sol mutant flies were able to stabilize their flight with respect to visual landmarks and fly straight (i.e., establish optomotor balance; Wolf \& Heisenberg, 1986). The interpretation was that rol sol mutant flies are motion sensitive but lacked sensitivity to the direction of motion. This was demonstrated by performing the third critical experiment (Figure 4). After inversion of the feedback loop between behavior and environment, such that attempted left turns lead to a left turn of the environment and thus the visual impression of a right turn, rol sol mutants did not require any more time a

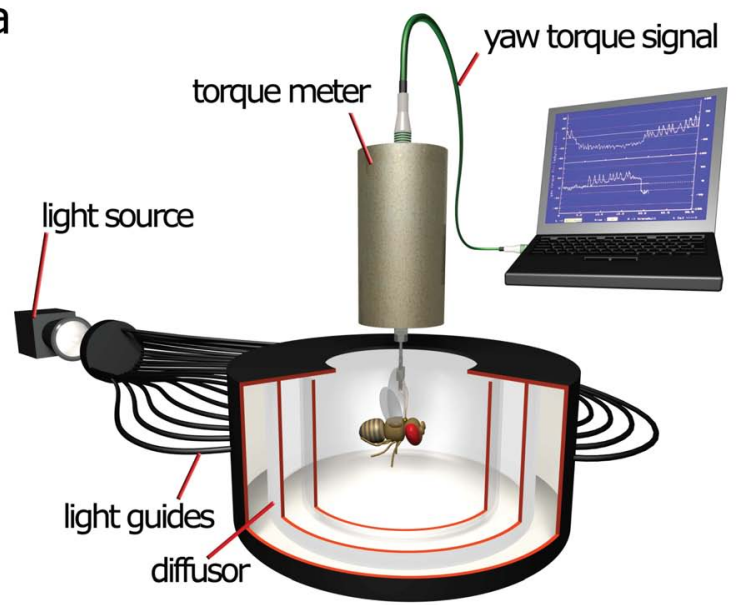

b
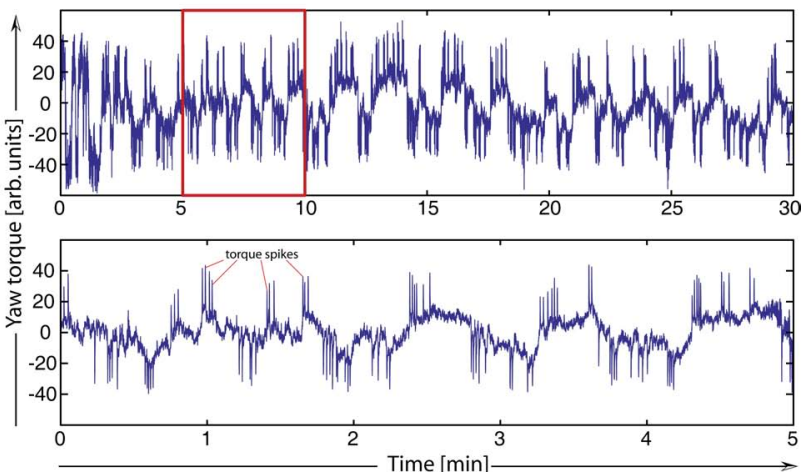

Figure 2. Measuring fruit fly spontaneity in a uniform, constant environment. (A) The fly is flying stationarily in a cylindrical arena homogeneously illuminated from behind. The fly's tendency to perform left or right turns (yaw torque) is measured continuously and fed into the computer. (B) Example of yaw torque data. Lower trace is a 5-minute enlargement of the 30minute upper trace. Variability in two components of the behavior can be observed: slow baseline fluctuations and fast, superimposed torque spikes. Torque spikes correspond to body saccades in free flight.

to stabilize their flight and fly straight than when then loop was closed "correctly." The conclusion that flies are actively initiating activity in order to "try out" which motor output controls the environment was confirmed when wild-type flies were subjected to this "inverting goggles" experiment. Even wild-type flies, with their optomotor response intact, eventually learned to use turning maneuvers of the "opposite" direction to control flight, that is, left-turning maneuvers for the visual impression of right turns and vice versa (Heisenberg and Wolf, 1984; Wolf et al., 1992). These three experiments attacked contemporary control theory from two ends: not only was output not predictable from input, but eliminating the open-loop response or inverting its direction could be compensated by plasticity in the system, such that it would still perform the function in question. Not only were the open loop situations an 


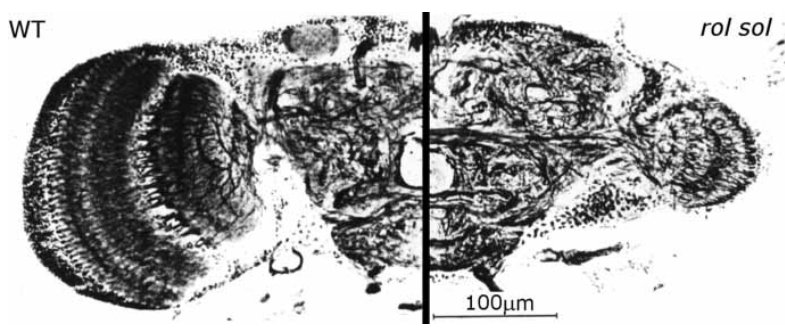

Figure 3. Frontal sections of wild-type (WT) and rol sol mutant brains. In rol sol flies, the optic lobes have about $12 \%$ of the wild-type volume. The remaining structures are retinotopically organized with the normal number of ommatidia in the eye and columns in the neuropil. Images courtesy of Martin Heisenberg.

inadequate experimental approach, but the input-output assumption itself proved inadequate as a theoretical construct for understanding even a fly brain. These experiments prompted Heisenberg to abandon this pervasive, successful approach and pursue a radically different research direction: how animals use their capacity for initiating output to control their sensory input. Today, 30 years later, more and more evidence - not only from neuroscience - is accumulating that while computing input-output relations may be an important feature of

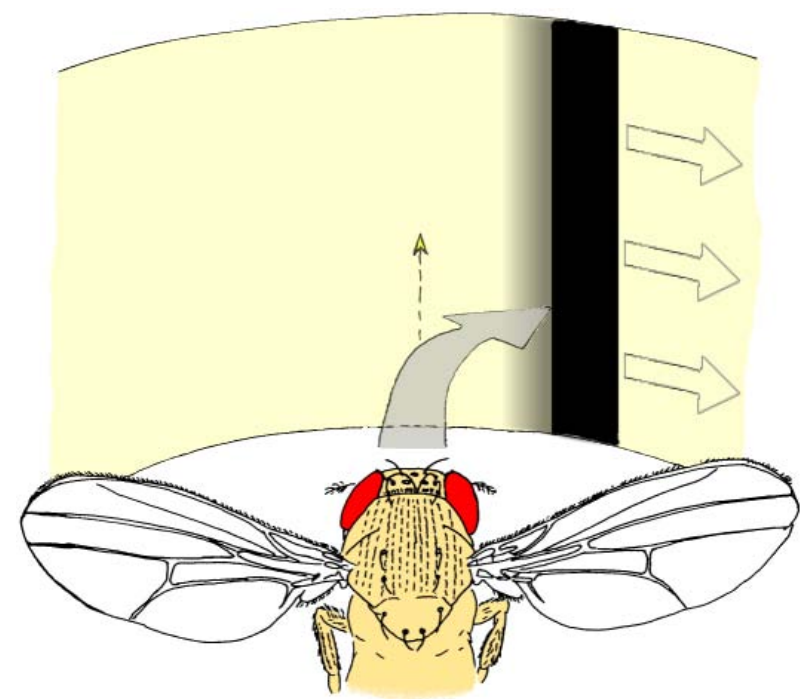

Figure 4. "Inverting goggles" experiment. Whenever the tethered fly attempts a turning maneuver, the fly's visual panorama is rotated in the same direction. In the depicted example, a right-turning maneuver leads to a rotation of the panorama to the right. In this situation, any attempts of the fly to follow the stripe will lead to a catastrophic feedback of increasing speed of the stripe and yaw torque of the fly in the same direction. Nevertheless, flies learn to generate turning maneuvers in the opposite direction in order to establish a zero net rotation of the stripe (optomotor balance). Fly drawing courtesy of Reinhard Wolf. brains, computing such output-input relations (e.g., in socalled forward models; Webb, 2004) is probably equally, if not more, fundamental to the organization of behavior. In the remainder of this paper, I will try to provide a short sketch of the literature today that is supporting Heisenberg's early insight.

If we allow ourselves to anthropomorphize, Heisenberg's observation that flies with a constant stimulus situation still produce variable behaviors may not be all that surprising. We have all experienced how difficult it is to stay absolutely still, maybe even how horrible it feels to be forced not to move. We want to move and we want to decide what body part to move when and where the movement should go. We all feel the very basic notion that we possess a certain flexibility in our choices (Montague, 2008). Bereaving humans of such freedom is frequently used as punishment, and the bereft do invariably perceive this limited freedom as undesirable. This experience of freedom is an important characteristic of what it is like to be human. It stems, in part, from our ability to behave variably. Voltaire expressed this intuition in saying, "Liberty then is only and can be only the power to do what one will" (Voltaire, 1752/1924). However the concept that we can decide to behave differently, even under identical circumstances, underlies not only our justice systems, but our electoral systems, educational systems, parenting, and, basically, all other social systems also presuppose behavioral variability and at least a certain degree of freedom of choice. Games and sports would be predictable and boring without our ability of constantly changing our behavior in always the same settings. Faced with novel situations, humans and most animals increase their behavioral variability (Bunzeck and Duzel, 2006; Roberts and Gharib, 2006; Shahan and Chase, 2002). Animals even vary their behavior when a more stereotyped behavior would be more efficient (Krechevsky, 1937). Inasmuch as behavioral variability between individuals has genetic components, it is a crucial factor of niche exploitation in evolution. Moreover, behavioral variability within individuals has been shown to be ecologically advantageous in game theoretical studies (Brembs, 1996; Glimcher, 2003, 2005; Glimcher and Rustichini, 2004; McNamara et al., 2004; Platt, 2004), in pursuit-evasion contests such as predatorprey interactions ("protean strategy") (Driver and Humphries, 1988; Grobstein, 1994; Miller, 1997; Shultz and Dunbar, 2006), in exploration and foraging (Belanger and Willis, 1996; Viswanathan et al., 1999), in mobbing attack patterns by birds, and in the variation of male songbirds' songs (Neuringer, 2004). Clearly, invariable behavior will be exploited (Jablonski and Strausfeld, 2000, 2001; Miller, 1997) and leaves an organism helpless in unpredictable situations (Greenspan, 2005; Heisenberg, 1994). 
Thus, competitive success and evolutionary fitness of all ambulatory organisms rely critically on intact behavioral variability as an adaptive brain function. But, relative freedom from environmental contingencies is a necessary, but most often not a sufficient, criterion for such accomplishments. Tightly connected to the ability to produce variable behavior is the ability to use the effects of these behaviors to control the environment. The incoming stream of sensory information is noisy and fluctuates for any number of reasons. Any covariance between the behavioral variations and those of sensory input indicates that the latter are consequences of the behavior and can thus be controlled be the animal (Bays et al., 2006; Wegner, 2002). Every animal relies heavily on this on-line detection system for when the animal itself is the reason for any environmental fluctuation. This function is so paramount that we humans express our delight over control of our environment (including other people) already as children, by, for example, shrieking in excitement when Dad jumps after a "boo" or proudly presenting Mom with "look what I can do!'. Later, children find pleasure in building airplane models, become carpenters with a delight for shaping wood, artists feeling gratified creating art out of the simplest materials, musicians enjoying mastering their instrument to perfection, athletes, scientists, engineers, managers, or cunning politicians. Using trial and error, we have shaped our world from caves to skyscrapers, from horses to jet planes, from spears to hydrogen bombs. Cultural or religious rituals (e.g., rain dance) and superstition may have developed as a means to create a feeling of control where, ultimately, there is none. Clearly, behaving flexibly in order to control our environment is at the heart of human nature and probably affects more aspects of our daily lives than any other brain function. So essential is such functioning that even very simple brains possess it. Even Drosophila prefers a situation in which it controls its environment over one where it does not. If certain flight directions are experimentally superimposed with uncontrollable visual movements, flies quickly avoid such directions and fly only in areas of full control (Heisenberg et al., 2001). This experiment demonstrates that control over environmental stimuli is inherently rewarding already for numerically simple, but very likely also for all other, brains. The same experiment also helps to understand how the rol sol mutants managed to fly straight: The flies spontaneously varied their motor output ("trying out") until they could control absolute movement (i.e., independently of direction) in their environment. Obviously, rol sol mutants are using an operant strategy to control their stimulus situation (Wolf and Heisenberg, 1986). The same strategy also must underlie the capacity of wild-type flies to master the "inverting goggles" experiment.
By detecting what component of the sensory stream is controlled by our own actions, operant behavior also underlies the distinction between observing and doing (i.e., differentiating between self and non-self). It is thought that one of the main principles behind operant behavior is the so-called reafference principle (Todorov, 2004; von Holst and Mittelstaedt, 1950; Webb, 2004). We compare our behavioral output (efference) with incoming sensory input (afference) to detect when we are the ones authoring environmental change (Bays et al., 2006; Wegner, 2002; Wolf and Heisenberg, 1991). One almost iconographic example of such behavior is to perform various spontaneous movements in front of a mirror to detect whether it is us we are perceiving. Even animals perform such movements in front of a mirror (Plotnik et al., 2006; Prior et al., 2008; Reiss and Marino, 2001). This automatic detection mechanism explains why we cannot tickle ourselves (Bays et al., 2006), why we perceive a stable visual world despite our frequent quick, or saccadic, eye movements (Sommer and Wurtz, 2006), and is reflected in different brain-activation patterns between self-generated and exogenous visual stimulation (Matsuzawa et al., 2005). It is thought that the detection is accomplished via an efference copy (or corollary discharge) of the motor command, which is compared to incoming afferent signals to distinguish re- from exafference. Such a differentiation has been implied to demonstrate causal reasoning in rats (Blaisdell et al., 2006; Clayton and Dickinson, 2006; Waldmann et al., 2006). Even robots can use such "self-modeling" to generate a continuously updated model of themselves and their environment (Bongard et al., 2006). The brain, then, uses this model to predict the sensory consequences of behavior, and the integration of this prediction with the actual sensory information is used to produce an estimate of sensory space that is enhanced over predictions from either ex- or reafferent stimulation alone (Vaziri et al., 2006). This effect of operant enhancement of sensory cues can be observed also in the fruit fly (Brembs and Plendl, 2008; Heisenberg et al., 2001), monkey (Kornell and Terrace, 2007), human (James, 1890; Slamecka and Graf, 1978), and robot (Gutnisky and Zanutto, 2004b) learning and may explain why starlings, but not tamarin monkeys, can recognize patterns defined by so-called recursive grammar (Marcus, 2006). Such control of sensory input has often been termed "goal-directed", behavior or action. At its basis lies the capacity to generate spontaneous variability: initiating activity. This perspective provides an intuitive understanding of the rewarding properties of being in control of the environment. Setting and obtaining goals is inherently rewarding (Kim et al., 2006). This reward ensures that individuals always actively strive to control. Expecting sensory feedback signals can go so far that willing to move a limb can lead to the illusion of limb movement, even if 
none occurred (Gandevia et al., 2006). One may also say that we so want our actions to have an effect that we sometimes develop a bad conscience even when we have not done anything wrong.

At the same time, by controlling the environmental input by using operant feedback loops, individuals exert their effect not only on themselves, but their survival and procreation in the environment they shape for themselves directly affects evolution. This has been shown in the field, for example, for western bluebirds, which dissociate into different niches according to their level of aggression (Duckworth, 2006). In humans, such mechanisms have been proposed to explain otherwise hard-to-understand phenomena, such as high IQ heritability estimates and associated paradoxa (i.e., increasing IQ heritability with age/experience and the "Flynn effect" of increasing IQ over generations) (Dickens and Flynn, 2001; Toga and Thompson, 2005). Another good example is the evolution of brain size. Most inter- and intraspecific interactions can be conceptualized as pursuit-evasion contests (e.g., predator/prey, male/female, dominant/subordinate, etc.). There are two reports on such contests leading to increased brain size. The first details how small-brained prey are more likely to be caught by predators, presumably because their capacity for behavioral variability is also smaller (Shultz and Dunbar, 2006). The second shows that the largest relative brain sizes among primate species are associated with monogamous mating systems, raising the suspicion that unpredictable mating strategies are the most successful ones in monogamous species (Schillaci, 2006). Other research in birds ties the evolution of brain size both to behavioral variability and migration: birds with larger brains are both more likely to be sedentary and cope better in novel environments. The hypothesis here is that a sedentary lifestyle in seasonally changing habitats requires significant behavioral flexibility. Operant feedback provides flexible birds with more resources, which enable them to support larger brains, which, in turn, generate more behavioral variability: Brain size and behavioral flexibility coevolved to outcompete other, smaller-brained birds that migrate in order to survive (Pravosudov et al., 2007; Sol et al., 2005a,b). Thus, the interdependence of brain size, the level of behavioral variability it provides, and the energy supply by which it is constrained are starting to unravel.

\section{CONCLUSIONS}

With this short overview, I hope to have shown that without initiating activity, there would not be any brains for us to study. Such spontaneous activity is both a necessary prerequisite and an inevitable consequence of evolution. Ultimately, the conceptually simple process of generating activity and evaluating its consequences forms one of the fundamental cornerstones not only for all of our human nature, but also for our social coherence: human nature as described in planning, willing, and controlling our behavior (Frith et al., 1991; Knight et al., 1995; Lezak, 1995; Owen, 1997; Wegner, 2002) and our social coherence, as based on cooperation (Gutnisky and Zanutto, 2004a; McNamara et al., 2004; Sanabria et al., 2003). Martin Heisenberg is a visionary and a pioneer in the neurobiological study of these and related neural processes. Only recently has his view of the primarily active nature of brains started to gain a more widespread acceptance among biologists.

\section{ACKNOWLEDGMENTS}

The author would like to thank Martin Heisenberg for his continued support and encouragement, for sharing his knowledge, experience, and wisdom, and for his way of leading by example.

Declaration of interest: The author reports no conflict of interest. The author alone is responsible for the content and writing of this paper.

\section{REFERENCES}

Bays, P. M., Flanagan, J. R. \& Wolpert, D. M. (2006). Attenuation of self-generated tactile sensations is predictive, not postdictive. PLoS Biol, 4(2), e28.

Belanger, J. H. \& Willis, M. A. (1996). Adaptive control of odor-guided locomotion: behavioral flexibility as an antidote to environmental unpredictability. Adapt Behav, 4(34), 217-253.

Blaisdell, A. P., Sawa, K., Leising, K. J. \& Waldmann, M. R. (2006). Causal reasoning in rats. Science, 311(5763), 1020 1022 .

Bongard, J., Zykov, V. \& Lipson, H. (2006). Resilient machines through continuous self-modeling. Science, 314(5802), 1118-1121.

Brembs, B. (1996). Chaos, cheating, and cooperation: potential solutions to the Prisoner's Dilemma. Oikos, 76(1), 14-24.

Brembs, B. \& Plendl, W. (2008). Double dissociation of PKC and $\mathrm{AC}$ manipulations on operant and classical learning in Drosophila. Curr Biol, 18(15), 1168-1117.

Bunzeck, N. \& Duzel, E. (2006). Absolute coding of stimulus novelty in the human substantia nigra/VTA. Neuron, 51(3), 369-379.

Clayton, N. \& Dickinson, A. (2006). Rational rats. Nat Neurosci, 9(4), 472-474.

Dickens, W. T. \& Flynn, J. R. (2001). Heritability estimates versus large environmental effects: the IQ paradox resolved. Psychol Rev, 108(2), 346-369.

Driver, P. M. \& Humphries, N. (1988). Protean behavior: the biology of unpredictability. Oxford, England: Oxford University Press. 
Duckworth, R. (2006). Aggressive behaviour affects selection on morphology by influencing settlement patterns in a passerine bird. Proc. R. Soc. Lond. B, 273(1595): 1789-95.

Frith, C. D., Friston, K., Liddle, P. F. \& Frackowiak, R. S. (1991). Willed action and the prefrontal cortex in man: a study with PET. Proc R Soc Lond B Biol Sci, 244(1311), 241-246.

Gandevia, S. C., Smith, J. L., Crawford, M., Proske, U. \& Taylor, J. L. (2006). Motor commands contribute to human position sense. J Physiol (Lond), 571(3), 703-710.

Glimcher, P. (2003). Decisions, Uncertainty, and the Brain: the Science of Neuroeconomics. Cambridge, Massachusetts, USA: MIT.

Glimcher, P. W. (2005). Indeterminacy in brain and behavior. Annu Rev Psychol, 56, 25-56.

Glimcher, P. W. \& Rustichini, A. (2004). Neuroeconomics: the consilience of brain and decision. Science, 306(5695), 447-452.

Götz, K. G. (1964). Optomoter studies of the visual system of several eye mutants of the fruit fly. Drosophila. Kybernetik, $2,77-92$.

Götz, K. G (1965). The optical transfer properties of the complex eyes of Drosophila. Kybernetik, 2, 215-221.

Götz, K. G. (1968). Flight control in Drosophila by visual perception of motion. Kybernetik, 4, 199-208.

Götz, K. G. (1972). Principles of optomotor reactions in insects. Bibl Ophthalmol, 82, 251-259.

Götz, K. G. (1980). Visual guidance in Drosophila. Basic Life Sci, 16, 391-407.

Götz, K. G., \& Buchner, E. (1978). Evidence for one-way movement detection in the visual system of Drosophila. Biol Cybern, 31, 243-248.

Götz, K. G., Hengstenberg, B., \& Biesinger, R. (1979). Optomotor control of wingbeat and body posture in Drosophila. Biol Cybern, 35, 101-112.

Greenspan, R. J. (2005). No critter left behind: an invertebrate renaissance. Curr Biol, 15(17), R671-R672.

Grobstein, P. (1994). Variability in behavior and the nervous system. In Ramachandran, V. S. (Ed.), Encyclopedia of Human Behavior (Vol. 4, pp. 447-458). New York: Academic Press.

Gutnisky, D. A. \& Zanutto, B. S. (2004a). Cooperation in the iterated Prisoner's Dilemma is learned by operant conditioning mechanisms. Artif Life, 10(4), 433-461.

Gutnisky, D. A. \& Zanutto, B. S. (2004b). Learning obstacle avoidance with an operant behavior model. Artif Life, 10(1), 65-81.

Heisenberg, M. (1983). Initiale aktivität und willkürverhalten bei tieren. Naturwissenschaften, 20, 70-78.

Heisenberg, M. (1994). Voluntariness (willkürfähigkeit) and the general organization of behavior. Life Sci Res Rep, 55, $147-156$.

Heisenberg, M. \& Wolf, R. (1979). On the fine structure of yaw torque in visual flight orientation of drosophila-melanogaster. J Comp Physiol A Sens Neural Behav Physiol, 130(2), 113-130.

Heisenberg, M. \& Wolf, R. (1984). Vision in Drosophila. Genetics of Microbehavior. Berlin, Heidelberg, New York, Tokyo: Springer.
Heisenberg, M., Wolf, R. \& Brembs, B. (2001). Flexibility in a single behavioral variable of Drosophila. Learn Mem, 8(1), $1-10$.

Jablonski, P. G. \& Strausfeld, N. J. (2000). Exploitation of an ancient escape circuit by an avian predator: prey sensitivity to model predator display in the field. Brain Behav Evol, 56(2), 94-106.

Jablonski, P. G. \& Strausfeld, N. J. (2001). Exploitation of an ancient escape circuit by an avian predator: relationships between taxon-specific prey escape circuits and the sensitivity to visual cues from the predator. Brain Behav Evol, 58(4), 218-240.

James, W. (1890). The Principles of Psychology. New York: Holt.

Kim, H., Shimojo, S. \& O’Doherty, J. P. (2006). Is avoiding an aversive outcome rewarding? Neural substrates of avoidance learning in the human brain. PLoS Biol, 4(8), 233.

Kirschfeld, K. \& Reichardt, W. (1970). Optomotor experiments on Musca with linearly polarized light]. Z Naturforsch B, 25(2), 228.

Knight, R. T, Grabowecky, M. F, \& Scabini, D. (1995). Role of human prefrontal cortex in attention control. Adv Neurol, 66, 21-34; discussion 34-26.

Kornell, N. \& Terrace, H. S. (2007). The generation effect in monkeys. Psychol Sci, 18(8), 682-685.

Krechevsky, I. (1937). Brain mechanisms and variability II. Variability where no learning is involved. J Compar Physiol Psychol, 23, 139-160.

Lezak, M. D. (1995). Neuropsychological Assessment (3rd edn). New York: Oxford University Press.

Marcus, G. F. (2006). Language: startling starlings. Nature, 440(7088), 1117-1118.

Matsuzawa, M., Matsuo, K., Sugio, T., Kato, C. \& Nakai, T. (2005). Temporal relationship between action and visual outcome modulates brain activation: an fMRI study. Magn Reson Med Sci, 4(3), 115-121.

Mauk, M. D. (2000). The potential effectiveness of simulations versus phenomenological models. Nat Neurosci, 3(7), 649-651.

Maye, A., Hsieh, C.-H., Sugihara, G. \& Brembs, B. (2007). Order in spontaneous behavior. PLoS One, 2, e443.

McNamara, J. M., Barta, Z. \& Houston, A. I. (2004). Variation in behaviour promotes cooperation in the Prisoner's Dilemma game. Nature, 428(6984), 745-748.

Miller, G. F. (1997). Protean primates: The evolution of adaptive unpredictability in competition and courtship. In Whiten, A., \& Byrne, R. W. (Eds.), Machiavellian Intelligence II: Extensions and Evaluations (pp. 312-340). Cambridge: Cambridge University Press.

Montague, P. R. (2008). Free will. Curr Biol, 18(14), R584R585.

Neuringer, A. (2004). Reinforced variability in animals and people: implications for adaptive action. Am Psychol, 59(9), 891-906.

Owen, A. M. (1997). Cognitive planning in humans: neuropsychological, neuroanatomical, and neuropharmacological perspectives. Prog Neurobiol, 53(4), 431-450.

Platt, M. L. (2004). Unpredictable primates and prefrontal cortex. Nat Neurosci, 7(4), 319-320. 
Plotnik, J. M., de Waal, F. B. M. \& Reiss, D. (2006). Selfrecognition in an Asian elephant. PNAS, 103(45), 1705317057.

Poggio, T. \& Reichardt, W. (1973a). Considerations on models of movement detection. Kybernetik, 13(4), 223-227.

Poggio, T. \& Reichardt, W. (1973b). A theory of the pattern induced flight orientation of the fly Musca domestica. Kybernetik, 12(4), 185-203.

Poggio, T. \& Reichardt, W. (1976a). Nonlinear interactions underlying visual orientation behavior of the fly. Cold Spring Harb Symp Quant Biol, 40, 635-645.

Poggio, T. \& Reichardt, W. (1976b). Visual control of orientation behaviour in the fly. Part II. Towards the underlying neural interactions. $Q$ Rev Biophys, 9(3), $377-438$.

Pravosudov, V. V., Sanford, K. \& Hahn, T. P. (2007). On the evolution of brain size in relation to migratory behaviour in birds. Anim Behav, 73(3), 535-539.

Prior, H., Schwarz, A. \& Güntürkün, O. (2008). Mirror-induced behavior in the magpie (Pica pica): evidence of selfrecognition. PLoS Biol, 6(8), 202.

Reichardt, W. (1962). Nervous integration in the facet eye. Biophys J, 2, 121-143.

Reichardt, W. \& Poggio, T. (1975). A theory of the pattern induced flight orientation of the fly Musca domestica II. Biol Cybern, 18(2), 69-80.

Reichardt, W, \& Poggio, T. (1976). Visual control of orientation behaviour in the fly. Part I. A quantitative analysis. Q Rev Biophys, 9(3), 311-375, 428-338.

Reichardt, W. \& Wenking, H. (1969). Optical detection and fixation of objects by fixed flying flies. Naturwissenschaften, 56(8), 424-425.

Reichardt, W. E. (1965). Quantum sensitivity of light receptors in the compound eye of the fly Musca. Cold Spring Harb Symp Quant Biol, 30, 505-515.

Reiss, D. \& Marino, L. (2001). Mirror self-recognition in the bottlenose dolphin: a case of cognitive convergence. PNAS, 98(10), 5937-5942.

Roberts, S. \& Gharib, A. (2006). Variation of bar-press duration: where do new responses come from? Behav Proc, 72(3), 215-223.

Sanabria, F., Baker, F. \& Rachlin, H. (2003). Learning by pigeons playing against tit-for-tat in an operant Prisoner's Dilemma. Learn Behav, 31(4), 318-331.

Schillaci, M. A. (2006). Sexual Selection and the evolution of brain size in primates. PLOS ONE, 1(1), 62.

Shahan, T. A. \& Chase, P. N. (2002). Novelty, stimulus control, and operant variability. Behav Anal, 25(2), 175-190.

Shultz, S. \& Dunbar, R. (2006). Chimpanzee and felid diet composition is influenced by prey brain size. Biol Lett, 2(4), 505-508.
Slamecka, N. J. \& Graf, P. (1978). Generation effect - delineation of a phenomenon. $J$ Exp Psychol Hum Learn Mem, 4(6), 592-604.

Sol, D., Duncan, R. P., Blackburn, T. M., Cassey, P. \& Lefebvre, L. (2005a). Big brains, enhanced cognition, and response of birds to novel environments. Proc Natl Acad Sci U S A, 102(15), 5460-5465.

Sol, D., Lefebvre, L. \& Rodriguez-Teijeiro, J. (2005b). Brain size, innovative propensity, and migratory behaviour in temperate Palaearctic birds. Proc Royal Soc B Biol Sci, 272(1571), 1433-1441.

Sommer, M. A., \& Wurtz, R. H. (2006). Influence of the thalamus on spatial visual processing in frontal cortex. Nature, 444: 374-377.

Todorov, E. (2004). Optimality principles in sensorimotor control. Nat Neurosci, 7(9), 907-915.

Toga, A. W. \& Thompson, P. M. (2005). Genetics of brain structure and intelligence. Annu Rev Neurosci, 28, 1-23.

Vaziri, S., Diedrichsen, J. \& Shadmehr, R. (2006). Why does the brain predict sensory consequences of oculomotor commands? Optimal integration of the predicted and the actual sensory feedback. J Neurosci, 26(16), 4188-4197.

Viswanathan, G. M., Buldyrev, S. V., Havlin, S., da Luz, M. G., Raposo, E. P. \& Stanley, H. E. (1999). Optimizing the success of random searches. Nature, 401(6756), 911-914.

Voltaire. (1752/1924). Voltaire's Philosophical Dictionary (H. I. Wolf, Trans.). New York: Knopf.

von Holst, E., \& Mittelstaedt, H. (1950). Das reafferenzprinzip. Wechselwirkungen zwischen zentralnervensystem und peripherie. Naturwissenschaften, 37, 464-476.

Waldmann, M. R., Hagmayer, Y. \& Blaisdell, A. P. (2006). Beyond the information given: causal models in learning and reasoning. Curr Direct Psychol Sci, 15(6), 307-311.

Webb, B. (2004). Neural mechanisms for prediction: do insects have forward models? Trends Neurosci, 27(5), 278-282.

Wegner, D. M. (2002). The Illusion of Conscious Will. Boston: Bradford Books/MIT press.

Wehrhahn, C. \& Reichardt, W. (1973). Visual orientation of the fly Musca domestica towards a horizontal stripe. Naturwissenschaften, 60(4), 203-204.

Wolf, R. \& Heisenberg, M. (1986). Visual orientation in motionblind flies is an operant behavior. Nature, 323(6084), 154-156.

Wolf, R. \& Heisenberg, M. (1991). Basic organization of operant behavior as revealed in Drosophila flight orientation. J Compar Physiol A Sens Neur Behav Physiol, 169, 699-705.

Wolf, R., Voss, A., Hein, S. \& Heisenberg, M. (1992). Can a fly ride a bicycle? Discussion on natural and artificial low-level seeing systems. Philos Transact Royal Soc London Series B Biol Sci, 337(1281), 261-269. 